BULLETIN (New Series) OF THE

AMERICAN MATHEMATICAL SOCIETY

Volume 48, Number 4, October 2011, Pages 509-511

S 0273-0979(2011)01345-3

Article electronically published on June 14, 2011

\title{
COMMENTARY ON \\ "ON THE PARALLELIZABILITY OF THE SPHERES" \\ BY R. BOTT AND J. MILNOR AND \\ "ON THE NONEXISTENCE OF ELEMENTS OF \\ HOPF INVARIANT ONE" BY J. F. ADAMS
}

\author{
ANDREW RANICKI
}

Immediately following the commentary below, these previously published articles are reprinted in their entirety: R. Bott and J. Milnor, On the parallelizability of the spheres, Bull. Amer. Math. Soc. 64 (1958), Part 1: 87-89; and J. F. Adams, On the nonexistence of elements of Hopf invariant one, Bull. Amer. Math. Soc. 64 (1958), 279-282.

In the words of Heine, "Es ist eine alte Geschichte, doch bleibt sie immer neu"an old story which stays forever new. Our old/new story concerns the finite dimensional division algebras over the reals $\mathbb{R}$ and real vector bundles over the spheres $S^{n}$.

By definition, an $n$-dimensional division algebra is an $n$-dimensional real vector space $V$ with a bilinear multiplication $V \times V \rightarrow V$ without zero divisors; the multiplication need be neither commutative nor associative. The story starts with the purely algebraic 19th century theorems of Frobenius and Hurwitz. They proved that there are only four normed division algebras, namely $\mathbb{R}$ itself with $n=1$, the complex numbers $\mathbb{C}$ with $n=2$, the quaternions $\mathbb{H}$ with $n=4$, and the octonions (1) with $n=8$.

In 1935 Hopf [Ho] used topology to prove that if there exists an $n$-dimensional division algebra, then $n=2^{k}$ for some $k \geqslant 0$. For $n \geqslant 2$ an $n$-dimensional division algebra $V$ determines:

1. A non-trivial $n$-dimensional vector bundle $\eta(V)$ over $S^{n}$, called the Hopf bundle of $V$;

2. A trivialization of the tangent bundle $\tau_{S^{n-1}}$ of $S^{n-1}$, so that $S^{n-1}$ is parallelizable.

The Hopf invariant $H(f) \in \mathbb{Z}$ of a map $f: S^{2 n-1} \rightarrow S^{n}$ showed that the Hopf bundles $\eta(V)$ for $n=2,4,8$ are nontrivial, since they determine maps $f$ with $H(f)=1$. The nontriviality of $\eta(V)$ also follows from the nontriviality of the mod 2 Stiefel-Whitney class $w_{n}(\eta(V)) \neq 0$.

Received by the editors May 25, 2011.

2010 Mathematics Subject Classification. Primary 57R22, 17A35.

(C)2011 American Mathematical Society Reverts to public domain 28 years from publication 
The first of my two selections is the 1958 announcement by Raoul Bott and John Milnor [BoMi] that the following conditions on $n \geqslant 1$ are equivalent:

1. There exists an $n$-dimensional division algebra;

2. There exists an $n$-dimensional vector bundle over $S^{n}$ with $w_{n} \neq 0$;

3. $S^{n-1}$ is parallelizable;

4. $n=1,2,4$, or 8 .

The full account was published in Milnor [Mi]. Kervaire $\mathrm{Ke}$ proved the nonparallelizability of $S^{n-1}$ for $n>8$ independently. (It is somewhat unfortunate that the paper [Ke] is not listed in the Mathematical Reviews.) Both proofs made use of the Bott periodicity theorem, which was still quite new then. Incidentally, it followed from the theorem that the $K$-groups of all real vector bundles over spheres are generated by the trivial bundles and the Hopf bundles.

The second of my two selections is the 1958 announcement by Frank Adams Ad1, that the following conditions on $n \geqslant 2$ are equivalent:

1. $S^{n-1}$ is an $H$-space, i.e., equipped with a continuous multiplication map $S^{n-1} \times S^{n-1} \rightarrow S^{n-1}$ with an identity;

2. There exists a map $f: S^{2 n-1} \rightarrow S^{n}$ with Hopf invariant $H(f)=1 \in \mathbb{Z}$;

3. $n=2,4$, or 8 .

The proof made use of secondary cohomology operations; the full account was published in Adams Ad2. The more elegant $K$-theoretic proof obtained a few years later by Adams and Atiyah [AdAt did not diminish the achievement of [Ad1].

Hirzebruch Hi has written a very readable account of the connections between division algebras and topology.

The two announcements are classic applications of algebraic topology to both algebra and topology, which changed the scenery.

The story continues! At the 2009 Edinburgh conference celebrating Michael Atiyah's 80th birthday, Hopkins announced the solution together with Hill and Ravenel HiHoRa of the 45-year-old Kervaire invariant problem: the title of the article is surely a deliberate echo of [Ad1, $\mathrm{Ad} 2$. In 1969 Browder $\mathrm{Br}$ used the Adams spectral sequence to prove that if there exists an $n$-dimensional framed (= stably parallelizable) manifold with Kervaire invariant 1 , then $n=2^{k}-2$. It is known that there exist such manifolds for $n=2,6,14,30,62$. The first three cases are realized by $S^{m} \times S^{m}(m=1,3,7)$ with the exotic framing determined by $\mathbb{C}, \mathbb{H}$ and $\mathbb{O}$. The result of HiHoRa states that $n=2^{k}-2 \leqslant 126$. The hunt is on to settle the remaining case, $n=126$.

\section{REFERENCES}

[Ad1] J. F. Adams, On the nonexistence of elements of Hopf invariant one, Bull. Amer. Math. Soc. 64 (1958), 279-282. MR0097059 (20:3539)

[Ad2] J. F. Adams, On the non-existence of elements of Hopf invariant one, Ann. of Math. (2) 72 (1960), 20-104. MR0141119 (25:4530)

[AdAt] J. F. Adams and M. F. Atiyah, K-theory and the Hopf invariant, Quart. J. Math. Oxford (2) 17 (1966), 31-38. MR0198460 (33:6618)

[BoMi] R. Bott and J. Milnor, On the parallelizability of the spheres, Bull. Amer. Math. Soc. 64 (1958), 87-89. MR0102804 (21:1590)

[Br] W. Browder, The Kervaire invariant of framed manifolds and its generalization, Ann. of Math. (2) 90 (1969), 157-186. MR0251736 (40:4963)

[HiHoRa] M. Hill, M. Hopkins and D. Ravenel, On the non-existence of elements of Kervaire invariant one, arXiv:0908.3724. 
[Hi] F. Hirzebruch, "Division algebras and topology", in Numbers, Graduate Texts in Mathematics 123, Springer-Verlag, New York, 1991, pp. 281-302.

[Ho] H. Hopf, Über die Abbildungen von Sphären auf Sphären niedriger Dimension, Fund. Math. 25 (1935), 427-440.

[Ke] M. Kervaire, Non-parallelizability of the $n$-sphere, $n>7$, Proc. Nat. Acad. U.S.A. 44 (1958), 280-283.

[Mi] J. Milnor, Some consequences of a theorem of Bott, Ann. of Math. (2) 68 (1958), 444-449. MR0102805 (21:1591)

UNIVERSITY OF EDINBURGH

E-mail address: a.ranicki@ed.ac.uk 